\title{
LIDAR MEASUREMENTS SUPPORTING THE OCULAR HAZARD DISTANCE CALCULATION USING ATMOSPHERIC ATTENUATION
}

\author{
K. Ove S. Gustafsson ${ }^{1 *}$, Rolf Persson ${ }^{1}$, Frank Gustafsson ${ }^{1}$, Folke Berglund ${ }^{1}$, Julia Hedborg ${ }^{1}$, \\ Jonas Malmquist ${ }^{2}$ \\ ${ }^{1}$ Swedish Defence Research Agency, P.O. Box 1165, SE-581 11 Linköping, Sweden, \\ *Email: ove.gustafsson@foi.se \\ ${ }^{2}$ Swedish Defence Materiel Administration, Vidsel Test Range, Box 74, SE-94 295 Vidsel, Sweden
}

\begin{abstract}
A series of lidar measurements has been performed at the Vidsel Test Range, Vidsel, situated in the inland of the very northern part of Sweden, as a part of an assessment of reducing the laser hazard distance using atmospheric attenuation within the calculations of nominal ocular hazard distance (NOHD). The question was "How low is the atmospheric attenuation as function of height in this area, using a wavelength of $1064 \mathrm{~nm}$ ?" The work included building a ground based backscatter lidar, performing a series of measurements and analyzing the results. The measurements were performed during June to November, 2014, with the objective to measure at clear air and good weather situations.
\end{abstract}

The lidar measurements at $1064 \mathrm{~nm}$ showed a very low atmospheric attenuation as a function of height to altitudes of at least $10 \mathrm{~km}$ at several occasions. The lowest limit of backscatter coefficient possible to measure with this instrument is $0.3 \cdot 10^{-7} \mathrm{~m}^{-1} \mathrm{sr}^{-1}$. Assuming a lidar ratio varying between $30-100 \mathrm{sr}$, this was leading to an extinction coefficient of about $0.9-3 \cdot 10^{-6} \mathrm{~m}^{-1}$. The atmospheric attenuation reduces the laser hazard distance with about $50-56 \%$ depending on the lidar ratio. A recommendation is to monitor the atmospheric attenuation at the occasions when the method to the reduced laser hazard distance using atmospheric attenuation is used.

\section{INTRODUCTION}

Lidar measurements were performed in an attempt to provide values on atmospheric attenuation as function of altitude for usage of powerful lasers at wavelength $1064 \mathrm{~nm}$ (Nd:YAG). The Lidar measured backscatter coefficient as function of height and standard values of lidar ratio was used to estimate the extinction coefficients. The ideal situation would have been using for instance three laser wavelengths measuring the backscatter coefficient and adding the information from two different Raman lidar signals, as suggested by Müller at al. [1] to calculate the extinction coefficient. Such system was not available and only the single backscatter was measured.

The distance where risk for injuries or damage of an eye when laser irradiance level exceeded the Maximum Permissible Exposure (MPE) is equal to the Nominal Ocular Hazard Distance (NOHD). The NOHD for lasers can be calculated using standardized methods as given by [2] and [3]. The NOHD is given, see [4], in the standard case by equation:

$$
N O H D=\frac{1}{\theta} \sqrt{\frac{4 P}{\pi M P E}-a^{2}}
$$

where $P$ is the laser power, $\theta$ the laser beam divergence and $a$ is the laser beam radius at the sender.

The NOHD for a typical designator laser with small divergence can be several tens of kilometers. As an example a designator laser, assuming a Nd:YAG laser, with wavelength 1064 $\mathrm{nm}$, power $0.180 \mathrm{~W}$, pulse length $15 \mathrm{~ns}$, PRF 11.5 $\mathrm{Hz}$ and beam divergence $0.08 \mathrm{mrad}$, will have NOHD of $48 \mathrm{~km}$ (multiple pulses during $10 \mathrm{sec}$ ).

One way of handling those risk distances, which might be too long for ordinary firing ranges or embargoed areas, are by probabilistic calculations of danger and by including the atmospheric attenuation. For such a long laser beam path the atmospheric transmission attenuation will be significant, depending on the level of attenuation. The suppression of the risk distance can be substantial even for moderate extinction coefficient if the atmospheric attenuation is included within the calculations of Ocular Hazard Distance (OHD).

The common way of calculating the NOHD abandons the use of atmospheric attenuation lowering the laser safety ranges in the civil society. The military community of interest has proposed 
several methods for exercises or testing taking into account the atmospheric attenuation under special conditions see [5] and [6]. A method to calculate the influence of a homogenous atmosphere on the laser safety range is given by [2]

$$
O H D_{\mu}=\frac{2 W\left(\frac{\mu(a+\theta r) \cdot \exp \left(\frac{\mu(a+\theta r)}{2 \theta}\right)}{2 \theta}\right)}{\mu}-\frac{a}{\theta}
$$

where $W$ is the Lambert W function, [7], $\mu$ is the extinction coefficient and $r$ is the beam radius.

The introduction of atmospheric attenuation in the calculation of the laser hazard distance could reduce the risk distance considerably, see Figure 1. The figure show the laser hazard distance as a function of visibility where a second scale of the abscissa gives the extinction coefficient of 1064 $\mathrm{nm}$ radiation. The dashed line show the NOHD for this particular laser system calculated with the standard method and the solid line shows the OHD when the atmospheric attenuation is taken into the account.

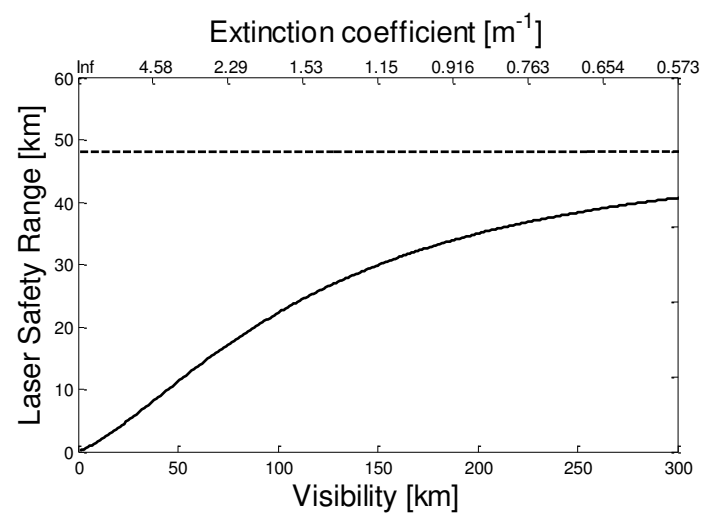

Figure 1. NOHD calculated by standard methods (dashed line) and OHD as function of visibility (solid line) for a designator laser using Nd:YAG laser.

The lidar system data, methodology of the lidar measurement and analysis of the signal is presented within chapter 2 together with a comparison between the $1064 \mathrm{~nm}$ lidar and our old $355 \mathrm{~nm}$ lidar system. In the comparison both system where measuring from the same place, within a $3 \mathrm{~m}$ distance. In chapter 3 examples of the results from the 2014 campaign is given. In the concluding chapter are the results discussed.

\section{METHODOLOGY}

A new lidar system was built up mostly from used equipment. A new detector with power supply unit and cooler support electronics was purchased from Licel $\mathrm{GmbH}$.

The laser is a Quantel Brilliant with pulse energy $230 \mathrm{~mJ}$ at $1064 \mathrm{~nm}$, pulse length $5 \mathrm{~ns}, \mathrm{PRF} 10 \mathrm{~Hz}$, beam divergence $0.5 \mathrm{mrad}$ and beam diameter 3 $\mathrm{mm}$. The laser beam is directed with a $45^{\circ}$ mirror to zenith. The beam is protected up to over eye level. See Figure 2.

The receiver telescope is constructed with hyperbolic mirrors, the primary mirror is $200 \mathrm{~mm}$ and the secondary mirror is $82 \mathrm{~mm}$ in diameter. The focal length is $615 \mathrm{~mm}$. Its axis is located 130 $\mathrm{mm}$ from the laser beam which gives full overlap well below $500 \mathrm{~m}$ from ground. The detector is an APD module from Licel GmbH (S11518-10) with a sensor diameter of $1 \mathrm{~mm}$ which gives a FOV of $1.6 \mathrm{mrad}$. The detector is thermoelectric cooled. A narrow filter with FWHM $12 \mathrm{~nm}$ decreases the background noise level.

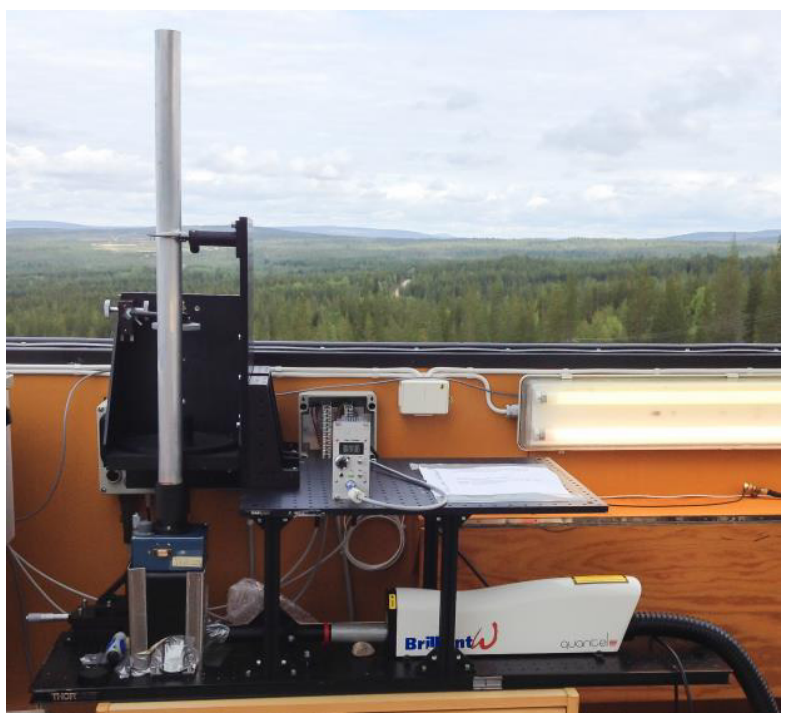

Figure 2. The $1064 \mathrm{~nm}$ lidar system was located in a small house with moveable roof for theodolite measurements.

Data Acquisition is performed with a Licel TR40 unit where 4000 bins are digitized at $40 \mathrm{MHz}$ sample rate. Usually 600 returns are averaged and saved as individual data files on a Windows computer during 30 minutes measurements. Measurement with covered laser beam during 15 minutes (laser warm up) gives 15 background 
files. The files were sent by the Lidar operator from Vidsel to FOI with email for analysis.

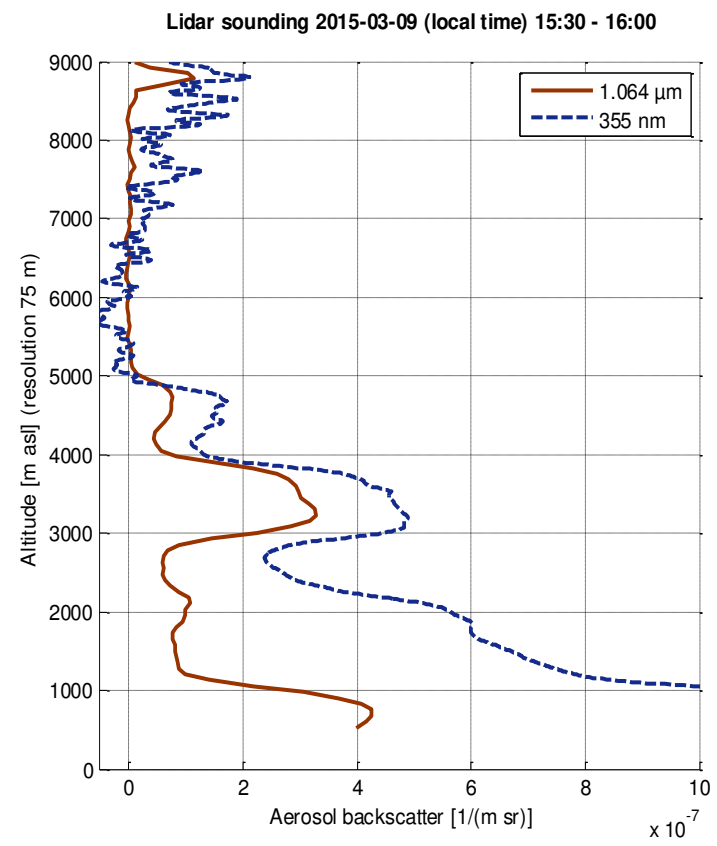

Figure 3. Backscatter levels from low levels up to $9 \mathrm{~km}$. Comparison between the new $1064 \mathrm{~nm}$ lidar and our old $355 \mathrm{~nm}$ lidar system.

The data files were viewed as "quicklook" graphs for data control and to make it convenient to eliminate bad measurements, e.g. clouds. Quicklooks are reachable at http://earlinet.foi.se/ (together with results from our previous engagements in Earlinet). Files sorted for further usage were analyzed with Klett's \& Fernald's methods [8] and [9]. Meteorological data for this analysis were achieved from SMHI (Swedish Meteorological and Hydrological Institute) as 1D-HIRLAM prognosis, calculated for the Lidar sites at Vidsel and Linköping four times every day.

The objective of the lidar measurements was to perform measurement at least once a week during six months, but only at occasions with very clean air, that means low amount of aerosol. The goal was not accomplished mainly since the weather and cloudiness did not fulfill the requirement of the campaign. Since the measurement was performed inside a test range, the range administration demanded that all measurement should be reported to the control tower in advance and the area had to be closed for flight operations. The laser risk range of the lidar laser is $11 \mathrm{~km}$.

\section{RESULTS}

During this period about 25 measurements were performed but only 16 could be completed and analyzed. At several occasions a measurement was started but too few profiles (due to clouds) could be collected to give a significant measurement. It was desired that at least 15 minutes of measurement, resulting in 15 profiles, were performed before the analysis was done. This was not always the case.

An example of measurement is shown in figure 4, where the number of profiles measured was lower than 15 but the number of aerosol layers looked very interesting. At least two layers are shown, one (at least) below $3 \mathrm{~km}$ and one above $7 \mathrm{~km}$. In between there is an altitude interval with very clean air.

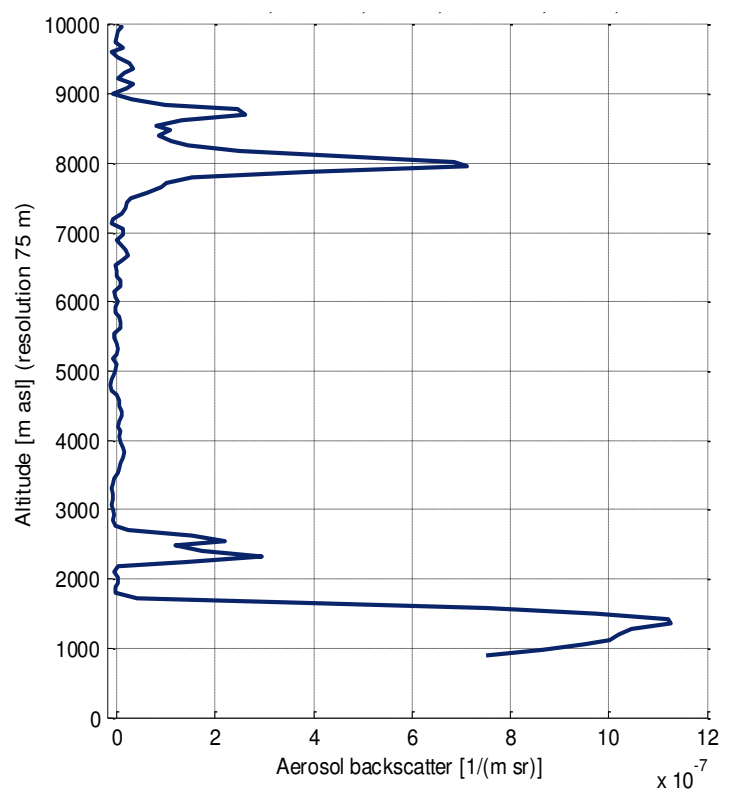

Figure 4. Lidar measurements at 09:30 UTC the 2014-09-10.

Since the purpose was different during this campaign than otherwise (looking for the situation with the lowest of aerosol content, lowest attenuation) the interest was focused on the clean days. Days with particular clean air were the June 30, September 1, and September 17. A lidar measurement from the September 17 is shown in figure 5. 


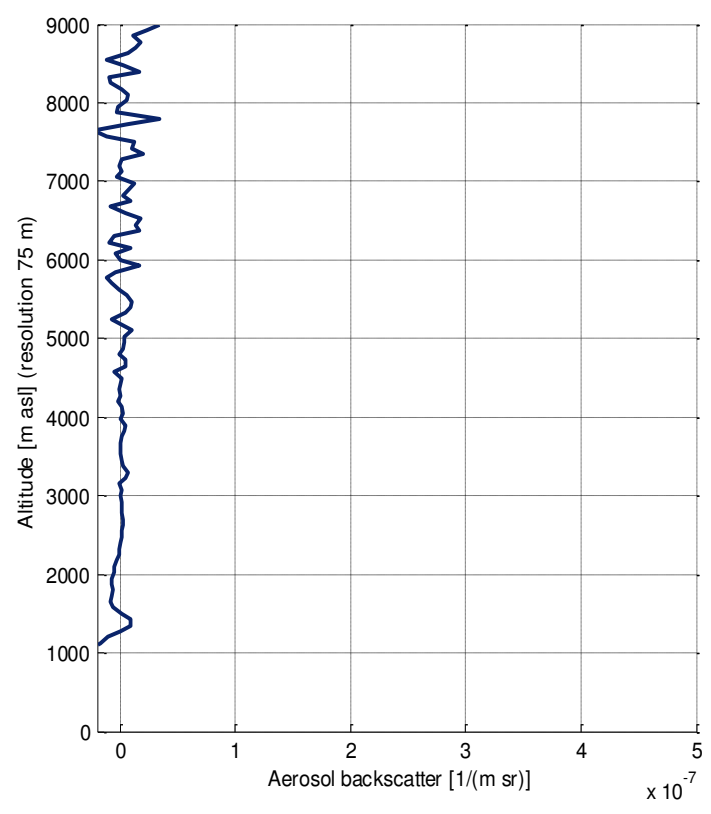

Figure 5. Lidar measurements at about 12:30 UTC the 2014-09-17.

The figure shows that the backscatter coefficient is lower than $0.3 \cdot 10^{-7} \mathrm{~m}^{-1} \cdot \mathrm{sr}^{-1}$. The backscatter extinction below zero is a consequence of background elimination and noise since values below zero does not exist in principle.

\section{CONCLUSIONS}

The objective was to find a lowest level of aerosol backscatter coefficient during his campaign, since the implication of low levels of extinction for this particular wavelength has very long laser hazards distances. The noise level did not give the opportunity setting a definitive lowest level of the aerosol extinction. The lowest possible measureable backscatter coefficient with this instrument was concluded to be less than $0.3 \cdot 10^{-7}$ $\mathrm{m}^{-1} \cdot \mathrm{sr}^{-1}$. This value does give an extinction value that depend of the type of aerosol to be $<0.9$ $3 \cdot 10^{-6} \mathrm{~m}^{-1}$, assuming a lidar ratio in the interval of $30-100$ sr. This leads to OHD values between 21 $-24 \mathrm{~km}$. This is a reduction of the NOHD with 50 - $56 \%$, depending on the lidar ratio assumption. The molecule extinction coefficient was assumed to be $5 \cdot 10^{-6} \mathrm{~m}^{-1}$, calculated by Modtran (program from Ontar Corporation) assuming no aerosol within the atmosphere.

The highest aerosol backscatter coefficient measured during this campaign was just above $2 \cdot 10^{-6} \mathrm{~m}^{-}$
${ }^{1} \cdot \mathrm{sr}^{-1}$, but again the objective was to find out the lowest values.

Reducing the NODH with atmospheric attenuation should be carried through with care and it is recommended to monitor the extinction coefficient during the activities.

\section{ACKNOWLEDGEMENT}

The author acknowledges the support from the Swedish Armed Force (FM) and Swedish Defence Material Administration (FMV), through Magnus Weideryd.

\section{REFERENCES}

[1] Müller D., Wandinger U., and Ansmann A., 1999: Microphysical particle parameters from extinction and backscatter lidar data by inversion with regularization: theory, Applied Optics, 38(12), 2346-2357.

[2] ANSI Z136.1-2014, 2014, [American National Standard for safe use of lasers].

[3] IEC 60825-1:2014, 2014 [Safety of laser products - Part 1: Equipment classification and requirements],.

[4] Gustafsson K. O. S., 2013: Questions about using of atmospheric attenuation calculating the nominal ocular hazard distance, Proc. of SPIE Vol. 8897, 88970M-1-8.

[5] JSP390 - "Military Laser Safety", 2005, UK Ministry of Defence - Ordnance Board

[6] STANAG 3606 - Edition 5, 1991, "Evaluation and Control of Laser Hazards".

[7] Steinvall O. K., 2009: Laser system range calculations and the Lambert $\mathrm{W}$ function, Applied Optics, 48(4), 1-7.

[8] Klett J. D., 1981: Stable analytical inversion solution for processing lidar returns, Applied Optics 20, 211-220.

[9] Fernald F. G., 1984: Analysis of atmospheric lidar observations: some comments, Applied Optics 23(5), 652-653. 